\title{
Alkaline peptone water enrichment with a dipstick test to quickly detect and monitor cholera outbreaks
}

Godfrey Bwire 1* D, Christopher Garimoi Orach², Dauda Abdallah³ ${ }^{3}$ Amanda Kay Debes ${ }^{4}$, Atek Kagirita ${ }^{5}$, Malathi Ram ${ }^{4}$ and David A. Sack ${ }^{4}$

\begin{abstract}
Background: Detection, confirmation and monitoring of cholera outbreaks in many developing countries including Uganda is a big challenge due to lack of the required resources and the time the test takes. Culture method which takes $24-48 \mathrm{~h}$ to get the feedback and requires highly skilled laboratory staff plus other complex resources is the standard test.

This study evaluated the new cholera rapid detection method that relies on Crystal VC dipsticks after enrichment with alkaline peptone water (APW) against the culture method for monitoring the progress of cholera outbreaks in rural setting.
\end{abstract}

Methods: We conducted the study between March and June 2015. Fresh stool samples and rectal swabs were incubated in $1 \%$ APW for $6 \mathrm{~h}$ at room temperature before testing with RDT following the manufacturer's instruction. The same stool sample was cultured to isolate $V$. cholerae in the standard manner. We also reviewed patient registers to epidemiologically describe the cholera epidemic.

Results: We tested stool from 102 consenting suspected cholera patients reporting during daytime at Bwera Hospital $(n=69)$, Kilembe Mines Hospital $(n=4)$ and Kinyabwama Health Centre $(n=29)$. Ninety one (91) samples were positive and nine samples were negative according to both methods. One (1) sample was positive only by dipstick and one sample was positive only by culture (sensitivity of 99\%, specificity of 90\%, Positive Predictive Value of 99\% and Negative Predictive Value of 90\%). Overall, 146 suspected cholera cases and two deaths, (case fatality rate of 1.36\%) were recorded during the study period. Among the cases aged 1-9 years, 63\% (50/79) were males while in those aged $20-49$ years, $76 \%$ (34/45) were females.

Conclusions: Our findings showed that the modified dipstick test after enrichment with 1\% APW had high level of accuracy in detection of $V$. cholerae and is quick, affordable alternative cholera outbreak monitoring tool in resource constrained settings. However, culture method should remain for cholera epidemic confirmation, for monitoring of antibiotic sensitivity and for production of pure isolates for molecular characterization. Further studies should be done to better understand the observed age and sex case distribution, in Kasese district.

Keywords: Vibrio cholerae, Rapid test, Dipstick, Alkaline peptone water, RDT, Outbreak detection, Epidemic monitoring

\footnotetext{
* Correspondence: cddmoh@yahoo.com

${ }^{1}$ Department of Community Health, Ministry of Health, Kampala, Kampala,

Uganda

Full list of author information is available at the end of the article
} 


\section{Background}

During the past four decades Uganda has experienced several cholera outbreaks in various regions of the country. Though cholera is a potentially preventable diarrheal disease, it continues to be a major public health problem in many rural districts in Uganda [1-6].

Cholera detection, confirmation and monitoring in Uganda and many other developing countries is by laboratory isolation of $V$. cholerae bacteria by culture method [7]. The culture method has several challenges namely; it takes 24-48 $\mathrm{h}$ to get the test results, requires highly skilled laboratory staff who may not be available in most rural areas in Africa [8], many supplies and good laboratory infrastructure particularly electricity $[9,10]$ to operate the equipments (incubators). There are only a few health facilities with the capacity to confirm cholera by culture in Africa; thus, samples are sent to a central laboratory whenever possible, but this takes additional time, sometimes weeks to obtain results [11]. If available and reliable, rapid diagnostic tests (RDTs) can facilitate the reporting, detection and monitoring of the progress of cholera outbreaks since they do not require complex infrastructure or highly skilled personnel, though some training is needed [12]. The RDTs also reduce the need for unnecessary laboratory tests and facilitate rapid implementation of appropriate infection control measures which is key in preventing infection spread [13].

The introduction of RDTs in malaria treatment in Uganda prevented misdiagnosis, improved patient care and saved government funds in medicine wastage associated with syndromic treatment of cases [14]. There is no similar reliable test for cholera and diagnosis is largely syndromic which is open to inclusion of patients with similar symptoms like cholera.

There are numerous challenges in detection and monitoring of cholera outbreaks that include long distances from the central laboratory (this laboratory is usually located in the capital city) to the cholera affected communities, inadequate laboratory supplies and lack of cholera specimen transport media among others. In addition, the normal practice after confirmation of the first 10-20 stool samples is to depend on syndromic diagnosis as recommended by World Health Organization [15]. This syndromic diagnosis is open to over or underestimation of cases in countries with high incidence of diarrhea such as Uganda where acute watery diarrhea is ranked $5^{\text {th }}$ major cause of morbidity and mortality [16].

Unfortunately, unlike for malaria and human immunodeficiency virus (HIV) infections, the currently available cholera rapid diagnostic testing methods are open to false positives which affect their reliability [17, 18]. Therefore, there is a need to improve and evaluate the accuracy of cholera RDTs to alert health workers in low resource countries to institute measures to prevent and control cholera outbreaks in timely manner.

This study evaluated a modification of the Crystal VC RDT test procedure after enrichment of stool sample in $1 \%$ alkaline peptone water (APW) against the culture method, the standard cholera confirmation and outbreak monitoring method in Uganda. Furthermore, the high $V$. cholerae detection level among the collected stool samples allowed for accurate epidemiological description of the outbreak in terms of the affected persons, place of origin, and the time of onset which provided a better understanding of factors responsible for the propagation of the epidemic in the population. This in return contributed to better cholera prevention and control efforts in the district.

\section{Methods}

\section{Study design}

We carried out a comparative study during a cholera outbreak that occurred between $14^{\text {th }}$ March and $26^{\text {th }}$ June 2015 in Kasese district, Western Uganda. We used a modified dipstick cholera RDT, Crystal VC, and compared it with the standard culture method for cholera detection, confirmation and monitoring which was available in this district. Crystal $V C$ is a trade name for the cholera testing dipsticks produced by Span Diagnostics Limited, Surat, India.

In addition, we reviewed information in the patient registers to epidemiologically describe the epidemic so as to guide cholera prevention and control activities in the district.

\section{Study area}

The study was conducted in Kasese district. According to Uganda Bureau of Statistics (UBOS), Kasese district had projected population derived from 2014 census of 702,029 persons [19]. The district is located in the remote part of Uganda. Kasese district shares a border with Eastern Democratic Republic of Congo (DRC), a border region known to have recurrent cholera outbreaks [20]. Suspected cholera cases seen at the three health facilities of Bwera Hospital, Kilembe Mines Hospital and Kinyabwamba Health centre III were enrolled in the study. Health facilities in Kasese district were purposively selected because previous studies indicated that Kasese was a district at high risk for cholera, with repeated cholera outbreaks [1, 21]. Furthermore, Kasese district had hospitals with the capacity to accurately carry out both the dipstick test and culture fecal specimens for cholera.

\section{Study population and inclusion criteria}

We only included admitted consenting suspected cholera patients seen in the three health facilities. For the patients to be admitted in the treatment facilities, the health workers 
used the national standard case definition for cholera that was adopted from WHO guideline for cholera [22]. In this guideline a suspected cholera case is defined as "in an area where the disease is not known to be present, a patient aged 5 years or more who develops severe dehydration or dies from acute watery diarrhea," or "in an area where there is a cholera epidemic (based on laboratory isolation of $V$. cholerae organisms and official declaration by the Ministry of Health, Kampala), the occurrence in a patient aged 2 years or more of acute watery diarrhea, with or without vomiting."

We excluded suspected cholera cases seen at the health facilities without capacity to carry out cholera testing on all reported cholera cases including culture confirmation and also excluded cases included in the register, but not meeting the standard case definition as per national cholera prevention and control guidelines.

\section{Stool collection procedures}

Stool samples were collected from patients following the national standard protocol for cholera stool sample collection [23]. Fresh stool samples or rectal swabs were collected from the patients by the health workers in the cholera treatment centre on arrival and before administration of antibiotic treatment and put in stool containers where they were immediately taken to the laboratory for testing and or culture. Laboratory testing was performed by trained personnel following the study protocol.

\section{Procedure for the enriched RDT and culture}

To conduct the RDT, the fresh stool sample (2 drops) or rectal swab was incubated in 1\% APW for $6 \mathrm{~h}$ at room temperature $\left(20-30{ }^{\circ} \mathrm{C}\right.$ with an average of $\left.25{ }^{\circ} \mathrm{C}\right)$ from which four drops of APW were drawn and put into a test-tube. Test was conducted following the manufacturer's instruction. To test for cholera, one Crystal VC RDT test strip was inserted into the test tube with $1 \%$ APW in vertical position and the test read after 10-15 min but not beyond $15 \mathrm{~min}$. The test reading was interpreted as negative, positive for O1, positive for $\mathrm{O} 1$ and $\mathrm{O} 139$, positive for $\mathrm{O} 139$ or invalid. The results were recorded immediately in the laboratory log book and on the data collection form which was sent back to the cholera treatment centre to serve as preliminary laboratory report. Patients found to be negative with the rapid test received the same recommended medical care as those who were positive for the test.

While incubating the stool samples in preparation for testing with the RDT the other portion of the stool sample was processed for cholera culture as outlined in the standard operation procedure for cholera identification [23]. After 24-48 h, the laboratory personnel read and recorded the culture results in the laboratory register and on the study forms. The culture results were also sent to cholera treatment centres as the final laboratory report.

\section{Quality control and further testing at Central Public Health Laboratory (CPHL)}

To ensure quality of the test results, every $10^{\text {th }}$ of positive sample had its isolate inoculated into a labeled Cary Blair transport media covered and packed into ice box before sending them to the Central Public Health Laboratory in Kampala to confirm the results for the purpose of quality control. Likewise, every $10^{\text {th }}$ of the negative samples were shipped to CPHL for quality testing and confirmation. All isolates were also sent to CPHL for further testing (serotyping) to ascertain the causative agent and serotyping. Serotyping was conducted using both polyvalent and monovalent antisera on fresh isolates following the national standard laboratory guidelines [23].

\section{Study variables, data storage and analysis}

Data was collected on age, sex, place of origin of the cholera cases, test results for enriched RDT test (positive, negative or invalid) and the culture results. The data was coded and stored on the spreadsheet. The data was analyzed to determine percentages, sensitivity, specificity, case fatality rates (CFR) and attack rates (AR). To calculate the attack rates we used population projection estimates for the sub-counties that were derived from 2014 national population and housing census conducted by Uganda Bureau of Statistics [19]. Data analysis was by use of spreadsheet and graphpad http://www.graphpad.com. The analysis was presented in tables and graphs. Spatial distribution of the cases and deaths was done using the shapefiles for administrative boundaries from Humanitarian Data Exchange website: https://data.humdata.org. The map showing the spatial distribution of cholera cases was produced using Arc GIS software; https://www.arcgis.com.

\section{Results}

\section{Laboratory results and interpretation}

Overall, 70\% (102/146) of suspected cholera cases seen during the study period, $14^{\text {th }}$ March to $26^{\text {th }}$ June 2015 had their stool tested using a modified RDT and the culture method. The laboratory results were as shown on Table 1.

Of the 92 samples positive by RDT, one was also positive for $V$. cholerae O139. This sample was sent to CPHL, Kampala and found to be positive for $V$. cholerae O1 Inaba. There were no V. cholerae O139 detected by culture. Further laboratory tests by serotypying of the $V$. cholerae isolates sent to CPHL, Kampala from Bwera Hospital laboratory showed that they were all $V$. cholerae O1 Inaba serotype. 
Table 1 Interpretation of 1\% APW enriched test and culture results for stool samples tested during the study period, $14^{\text {th }}$ March to $26^{\text {th }}$ June 2015

\begin{tabular}{llll}
\hline & \multicolumn{3}{l}{ Standard Diagnostic test (culture) } \\
\hline Enriched test with 1\% APW & Positive & Negative & Total \\
Positive & 91 & 1 & 92 \\
Negative & 1 & 9 & 10 \\
Total & 92 & 10 & 102 \\
Sensitivity & $98.9 \%,(95 \%$ Cl: 94.09-99.97\%) \\
Specificity & $90 \%,(95 \%$ Cl: 55.5-99.75\%) \\
Positive Predictive Value (PPV) & $98.9 \%$ & & \\
Negative Predictive Value(NPV) & $90.0 \%$ & & \\
\hline
\end{tabular}

\section{Quality control and serotyping results}

All ten (10) positive isolates sent for quality control testing in Kampala turned out positive for $V$. cholerae and the two negative specimens were negative for $V$. cholerae. Further serotyping with polyvalent (poly Inaba Ogawa) and monovalent Inaba showed positive for all isolates.

\section{Epidemiological description of the cholera outbreak}

The cholera outbreak in Kasese district started on $14^{\text {th }}$ March 2015 and the last suspected cholera case was admitted on $26^{\text {th }}$ June 2015. A total 146 cases and two deaths, (case fatality rate of $1.36 \%$ ) were reported during the study period in the three treatments centre of Bwera Hospital $n=107$, Kilembe Hospital $n=4$ and Kinyabwamba Health centre III $n=35$.

This cholera outbreak lasted for 16 weeks, had three peaks with the highest peak occurring during the $19^{\text {th }}$ calendar week (26 ${ }^{\text {th }}$ April-9 ${ }^{\text {th }}$ May 2015). The weekly number of reported cholera cases and deaths were as shown in Fig. 1.

During this outbreak there were two deaths. The first death occurred on $20^{\text {th }}$ April 2015 of a patient who was brought late at Bwera Hospital CTC and on examination was unconscious with severe dehydration and died few hours after admission despite intensive care from the medical team. The second death was a patient who was misdiagnosed and admitted in Bwera Hospital medical ward at night without undergoing full screening to exclude cholera. Given the inadequate monitoring at night on the medical ward, the patient deteriorated and died. Rectal swab and culture was done on the body after the death. Both the enriched RDT and culture were positive for $V$. cholerae. In response to these findings, Bwera Hospital management conducted an audit and took corrective action to prevent similar future scenario.

In this outbreak, all age groups and both sexes were affected. In the younger age group (1-19 years) there were more males $63 \%$ (50/79), but among young adults

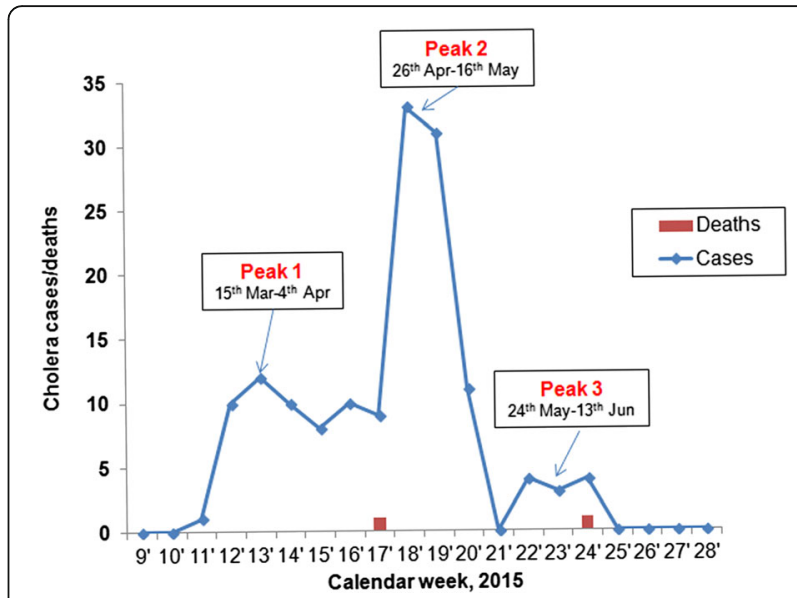

Fig. 1 Weekly reported cholera cases and deaths in the three cholera treatment centres in Kasese district, March-June 2015

(20-49 years) there were more females $76 \%(34 / 45)$. The age and sex of the cases were as shown in Fig. 2.

\section{Distribution of cholera cases by place of origin}

The 2015 projected population of Kasese, was 702,029 persons, but the cases came from 33\% (10/30) subcounties in the district. The affected sub-counties had a total projected population of 263,064 persons. The affected sub-counties with respective number of cases and cholera attack rates are shown in Table 2.

\section{Discussion}

Our study showed that the enriched dipstick test was able to rapidly detect cholera patients and simplify the

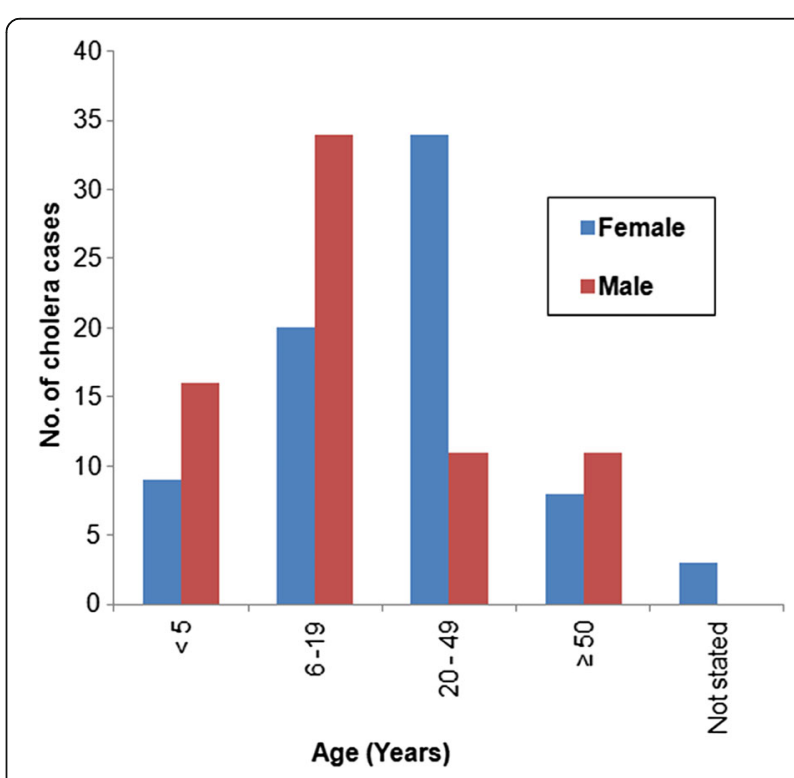

Fig. 2 Age and sex of the suspected cholera cases recorded in the three cholera treatment centres in Kasese district, March-June 2015 
Table 2 Cholera attack and case fatality rates stratified by the sub-counties of origin of cases during cholera outbreak in Kasese district, March-June 2015

\begin{tabular}{llllll}
\hline Place or Subcounty of Origin & Number of cases & Number of Deaths & Projected Population & Attack Rate Per 10,000 & Case Fatality Rate \\
\hline DRC & 5 & 0 & - & 0.0 & $0.0 \%$ \\
Ihandiro & 20 & 0 & 13,549 & 14.8 & $0.0 \%$ \\
Isango & 3 & 0 & 8099 & 3.7 & $0.0 \%$ \\
Karambi & 19 & 1 & 8099 & 23.5 & $5.3 \%$ \\
Katwe TC & 1 & 0 & 6411 & 1.6 & $0.0 \%$ \\
Bwera & 7 & 0 & 17,455 & 4.0 & $0.0 \%$ \\
MLTC & 28 & 1 & 51,018 & 3.5 & $0.6 \%$ \\
Mukunyu & 1 & 0 & 1854 & 0.3 & $0.0 \%$ \\
Maliba & 8 & 0 & 47,585 & 1.7 & $0.0 \%$ \\
Kitswamba & 31 & 0 & 30,009 & 0.3 & $0.0 \%$ \\
Nyakiyumbu & 13 & 0 & 31,854 & 17,131 & $0.0 \%$ \\
Kitholhu & 10 & 0 & 263,064 & 5.8 & $0.0 \%$ \\
Total & 141 & 2 & 5.4 & $1.4 \%$ \\
\hline
\end{tabular}

Note that five (5) patients came from the neighboring country, Democratic Republic of Congo (DRC)

Most of the cases came from the nearby sub-counties, close to the hospitals as shown in Fig. 3

monitoring of the outbreak progress in this remote part of Uganda. By using the enrichment method, the high sensitivity, specificity, positive and negative predictive values suggest that this test procedure provides reliable results for both outbreak detection and monitoring.

Furthermore, the test is affordable (each dipstick costing USD 1.9 compared to USD 4 for culture) [18], takes shorter time and can be done in rural typical African setting saving the health workers the costly sample transportation procedures.

This enriched dipstick test is a good alternative to the expensive culture method for monitoring of cholera outbreaks and is game changer for resource constrained countries like Uganda [24] with limited laboratory infrastructure, personnel and transportation challenges.

The significance of this innovation lies in the fact that, the key reagent (alkaline peptone water) required for this rapid test is part of the normal laboratory supplies and will not require special order and importation. Also Crystal $V C$ dipsticks are not new, they have been around for over two decades [17] and can easily be procured and stored. What is new is the technique of using the two components to detect and monitor cholera outbreaks more accurately and at lower or affordable cost by communities with inadequate funds and laboratory infrastructure.

It should be noted that Crystal VC dipsticks when used directly with stool, false positives have been reported commonly $[17,18]$ but when fecal specimens are first incubated in $1 \%$ APW, false positive results were found to be rare. The enriched dipstick method is not a bedside test, but results are generally available the same day as compared to 24-48 h required for culture method. Most importantly, the test is simple and can be performed even in remote areas without a microbiology laboratory or highly skilled technicians. These more reliable results enabled launching a rapid cholera alert and response in Kasese district. Besides being suitable for use in remote areas, the enriched test had several additional benefits.

First, the early cholera alert facilitated preparations for managing the outbreak within the district which led to relatively fewer cholera causes and shorter duration of the epidemic (16 weeks) compared to previous years (where outbreak lasted for over 9 months) [25].

Second, the dipstick test allowed the hospitals and the district staff to monitor the patients and the course of the outbreak more closely than ever before. There was quick action since laboratory results were received the same day. Unlike in the previous outbreaks where the normal practice was to transport random stool samples to Kampala or laboratory within the region and wait for 3-7 days to get laboratory report to guided the next steps [22], the test were done within the health facility and results received within a day.

Third, there was better use of laboratory reports such as recognizing that even during cholera outbreaks; some cases with diarrhea who met the standard case definition may be due to other causes and required immediate referral to other sections within the hospital.

Even though reliable test results were quicker to get and available the same day compared to culture method that took more days, health workers in Kasese district still asked for a shorter time period similar to that for malaria RDTs in which the time was 15-20 $\min [26,27]$.

The authors recommend that in order to strengthen the management of cholera in similar manner to other 
infectious diseases of public health importance such as malaria, a rapid and reliable diagnostic test should be an essential component of cholera prevention and control efforts.

Therefore, the use of the dipstick will not totally replace culture since culture is still needed to confirm epidemics, to monitor antibiotic sensitivity and to produce pure isolates for molecular characterization. However, the enriched dipstick method can be a valuable tool for detecting and monitoring cholera outbreaks.

Furthermore, epidemiological description of the outbreak using more reliable test highlighted important observations. First, although all ages were affected, we also noted that the younger age groups, up 19 years were the majority in the patient registers. Since this age group includes the school age, we think that cholera could contribute significantly to school absenteeism since studies on cholera in Kasese district showed that cholera was an endemic disease occurring frequently in some subcounties within the district [28].

Second, the high proportion of women in the reproductive years was an indication of increased risk for this age, sex group. Therefore, provision of targeted health education and the use of Oral Cholera Vaccines (OCV) for these groups and other eligible ones in endemic districts such as Kasese [28] could be explored as recommended by the current WHO guidelines [29]. Since global demand for OCV outweigh the needs [30] targeting the most at risk/ vulnerable groups namely; the children under 9 years and the women should result in maximum benefit from integrated cholera prevention and control interventions.

Third, though the case fatality rate was low among the patients in this study, the death that occurred while a patient was being treated on the medical ward emphasizes the need for careful observation and documentation of fluid losses for patients with severe watery diarrhea. The earlier use of the rapid test might have alerted the health personnel that the patient had cholera; however, it should be noted that cholera is not the only cause of severe diarrhea and rehydration treatment depends on the symptoms and not on the result of the rapid test.

Forth, it was worth noting that most of the cases detected in this study came from sub-counties close to the health facilities as shown in Fig. 3. Since cholera outbreaks

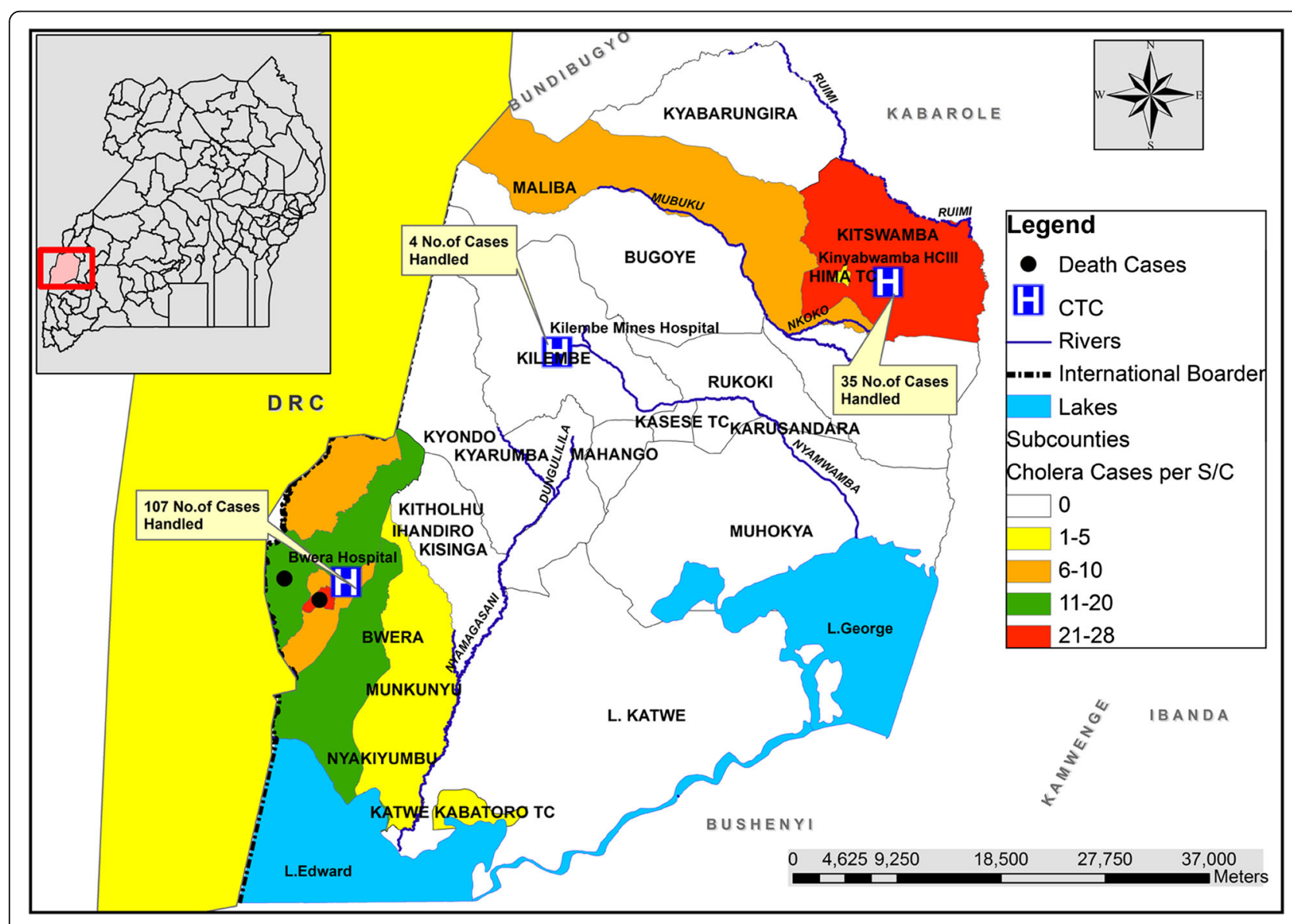

Fig. 3 The map of Kasese district showing distribution of cholera cases by their places of origin that were treated at the three cholera treatment centers, March-June 2015 
are rarely so focused near health facilities, it seems likely that these outbreaks also affected communities further away but may not have come to the designated cholera treatment centres or were treated but not reported.

The authors therefore recommend follow-up studies to determine the following: the source of the cholera outbreak; the explanation for the observed age and sex distribution to why more males below the age of 20 years were affected than the female counterparts and why more adult females were affected and a study on actual impact of cholera during an outbreaks such as the one describe in this study since the cases being treated at the health facility may represent a portion of the actual number of cases and deaths.

Finally, while results after $6 \mathrm{~h}$ and within a day was useful for decision making in remote areas, there is a need for rapid and a reliable test that provides results more quickly, similar to the malaria test which gives test results within $20 \mathrm{~min}[26,27]$.

\section{Conclusions}

Our findings showed that the modified RDT test after enrichment with $1 \%$ alkaline peptone water had high level of accuracy in detection of $V$. cholerae and is quick, affordable alternative cholera outbreak monitoring tool that should be put to wider use in resource constrained settings. However, culture method should remain for cholera outbreak/epidemic confirmation, to monitor antibiotic sensitivity and to produce pure isolates for molecular characterization.

\begin{abstract}
Abbreviations
AKD: Amanda Kay Debes; APW: Alkaline Peptone Water; AR: Attack rate; CFR: Case fatality rate; CGO: Christopher Garimoi Orach; CPHL: Central Public Health Laboratory; DAS: David A. Sack; DRC: Democratic Republic of Congo; GB: Godfrey Bwire; HIV: Human immunodeficiency virus; IRB: Institution Review Board; MLTC: Mpondwe-Lubirikha Town Council; MR: Malathi Ram; RDT: Rapid diagnostic test; TC: Town Council; UBOS: Uganda Bureau of Statistics; WHO: World Health Organization
\end{abstract}

\section{Acknowledgements}

We thank the health workers in Kasese district who collected the data and treated the patients; Makerere University School of Public Health for supervision and monitoring of the study; the staff of Central Public Health Laboratories, Kampala, for conducting the quality control tests and Mr. Ambrose Wabwire Buyinza for technical support in drawing of the epi-map.

We are grateful to Uganda Ministry of Health and the Bill and Melinda Gates Foundation for financial support.

\section{Funding}

The study was jointly funded by Uganda Ministry of Health and Bill and Melinda Gates Foundation working through the Delivering Oral Vaccine Effectively (DOVE) project (OPP153556), administered by the Johns Hopkins Bloomberg School of Public Health for Jointly funding the study. The funders had no role in the study design, data collection and analysis, decision to publish, or preparation of the manuscript

\section{Availability of data and materials}

Data set used/analyzed to generate the tables and the figures are available from the corresponding author on reasonable request.

\section{Authors' contributions}

Conceived the idea; GB, CGO, DAS: Supervised data collection; DA, GB, AK, CGO, MR, DAS: Analysed data and compiled the first draft; GB, AKD, DAS: Contributed to the writing of the final manuscript: GB, CGO, DA, AK, AKD, MR, DAS; Overall supervision; CGO, DAS: Agreed with the final manuscript and conclusions; GB, CGO, DA, AK, AKD, MR, DAS.

\section{Ethics approval and consent to participate}

Written informed consents were obtained from the study participants (patients suspected of having cholera) and parents or guardians for the patients below the age of 18 years. Confidentiality was observed at all levels of data collection, storage, data analysis and dissemination. Permission to conduct the study was obtained from the Makerere University School of Public Health Institutional Review Board (IRB 00011353).

\section{Consent for publication}

Written informed consent was obtained from all study participants (patients suspected of having cholera) and parents or guardians for the patients below the age of 18 years. Confidentiality was observed at all levels of data collection, storage, analysis and dissemination.

\section{Competing interests}

The authors declare that they have no competing interests.

\section{Publisher's Note}

Springer Nature remains neutral with regard to jurisdictional claims in published maps and institutional affiliations.

\section{Author details \\ ${ }^{1}$ Department of Community Health, Ministry of Health, Kampala, Kampala, Uganda. ${ }^{2}$ Department of Community and Behavioral Sciences, Makerere University School of Public Health, College of Health Sciences, Kampala, Uganda. ${ }^{3}$ Kasese District Local Government, Kampala, Uganda. ${ }^{4}$ Department of International Health, DOVE Project, Johns Hopkins Bloomberg School of Public Health, Baltimore, MD, USA. ${ }^{5}$ Uganda National Health Laboratory Services (UNHS/CPHL), Ministry of Health, Kampala, Uganda.}

Received: 16 May 2017 Accepted: 12 November 2017

Published online: 21 November 2017

\section{References}

1. Bwire G, Malimbo M, Maskery B, Kim YE, Mogasale V, Levin A. The Burden of Cholera in Uganda. Ryan ET, editor. PLoS Negl Trop Dis. 2013 [cited 2013 Dec 11];7(12):e2545. Available from: http://dx.plos.org/10.1371/journal.pntd. 0002545

2. Bwire G, Malimbo M, Makumbi I, Kagirita A, Wamala JF, Kalyebi P, et al. Cholera surveillance in Uganda: An analysis of notifications for the years 2007-2011. J Infect Dis [Internet]. 2013;208:S78-85. Available from: https:// academic.oup.com/jid/article-lookup/doi/10.1093/infdis/ijt203.

3. Legros D, McCormick M, Mugero C, Skinnider M, Bek'Obita DD, Okware SI. Epidemiology of cholera outbreak in Kampala, Uganda. East Afr Med J. 2000;77:347-9.

4. Cummings MJ, Wamala JF, Eyura M, Malimbo M, Omeke ME, Mayer D, et al. A cholera outbreak among semi-nomadic pastoralists in northeastern Uganda: epidemiology and interventions. Epidemiol Infect. 2012;140:1376-85.

5. Alajo SO, Nakavuma J, Erume J. Cholera in endemic districts in Uganda during El Nino rains: 2002-2003. Afr Health Sci. 2006;6(2):93-7.

6. Bwire G, Malimbo M, Kagirita A, Makumbi I, Mintz E, Mengel MA, et al. Nosocomial cholera outbreak in a mental hospital: challenges and lessons learnt from butabika national referral mental hospital, Uganda. Am J Trop Med Hyg. 2015;93(3):534-8.

7. Kasolo F, Yoti Z, Bakyaita N, Gaturuku P, Katz R, Fischer JE, et al. IDSR as a platform for implementing IHR in African countries. Biosecur Bioterror. 2013; 11(3):163-9. Available from: http://www.pubmedcentral.nih.gov/articlerender. fcgi?artid=3779000\&tool=pmcentrez\&rendertype $=$ abstract

8. Fieno JV, Dambisya YM, George G, Benson K. A political economy analysis of human resources for health (HRH) in Africa. Hum Resour Health. 2016;14(1):44.

9. Eberhard A, Foster V, Briceño-Garmendia C, Ouedraogo F, Camos D, Shkaratan M. Underpowered State of the Power Sector in Sub-Saharan Africa [Internet]. Background Paper 6, Africa Infrastructure Country Diagnostic. 2008. 54 p. 
Available from: https://openknowledge.worldbank.org/bitstream/handle/ 10986/7833/482140ESWOP11110PowerOSectorOReview.pdf?sequence $=1$

10. Adair-rohani H, Zukor K, Bonjour S, Wilburn S, Kuesel AC, Hebert R, et al. Limited electricity access in health facilities of sub-Saharan Africa : a systematic review of data on electricity access, sources, and reliability. Glob Heal Sci Pract. 2013;1(2):249-61.

11. Petti $C$ a, Polage $C R$, Quinn TC, Ronald $A R$, Sande $M$ a. Laboratory medicine in Africa: a barrier to effective health care. Clin Infect Dis. 2006;42(3):377-82.

12. Counihan H, Harvey SA, Sekeseke-Chinyama M, Hamainza B, Banda R, Malambo T, et al. Community health workers use malaria rapid diagnostic tests (RDTs) safely and accurately: results of a longitudinal study in Zambia. Am J Trop Med Hyg. 2012;87(1):57-63.

13. D'Acremont V, Greub G, Genton B. Rapid diagnostic tests (RDT): the cure-all for the practitioner? Rev Med Suisse. 2011;7(294):984-6. 990

14. Kyabayinze DJ, Asiimwe C, Nakanjako D, Nabakooza J, Counihan H, Tibenderana JK. Use of RDTs to improve malaria diagnosis and fever case management at primary health care facilities in Uganda. Malar J. 2010;9:200.

15. WHO and CDC. Technical Guidelines for Integrated Disease Surveillannce and Response in the African Region. 2010;1-416. Available from: http:// www.healthdata.org/uganda.

16. Institute for Health Metrics and Evaluation WU. Health Data Uganda [Internet]. 2015 [cited 2017 May 5]. Available from: http://www.healthdata.org/uganda

17. Dick MH, Guillerm M, Moussy F, Chaignat C-L. Review of two decades of cholera diagnostics - how far have we really come? PLoS Negl Trop Dis. 2012;6(10):e1845. Available from: http://journals.plos.org/plosntds/article?id= 10.1371/journal.pntd.0001845

18. Ley B, Khatib AM, Thriemer K, von Seidlein L, Deen J, Mukhopadyay A, et al. Evaluation of a rapid dipstick (crystal VC) for the diagnosis of cholera in Zanzibar and a comparison with previous studies. PLoS One. 2012;7(5): e36930. Available from: http://dx.plos.org/10.1371/journal.pone.0036930.

19. Uganda Bureau of Statistics. National Population and Housing Census 2014. Uganda Bur Stat 2016, Natl Popul Hous Census 2014 - Main Rep, Kampala, Uganda [Internet]. 2014;1-209. Available from: http://www.ubos.org/ onlinefiles/uploads/ubos/NPHC/2014 National Census Main Report.pdf.

20. Muyembe JJ, Bompangue D, Mutombo G, Akilimali L, Mutombo a, Miwanda $\mathrm{B}$, et al. Elimination of cholera in the Democratic Republic of the Congo: the new National Policy. J Infect Dis. 2013;208(suppl 1):S86-91.

21. S.A. O. Comparison of two communities affected by cholera in Kasese district in Uganda. Am J Trop Med Hyg. 2013;89(5):205.

22. Ministry of Health Uganda. Prevention and control of cholera, operational guidelines for the district health workers and planners; 2007. p. 13-4.

23. Ministry of Health Uganda. Standard operational procedure for laboratory isolation of Vibrio cholera. 2006.

24. World Bank. World Development Indicators. 2017 [cited 2017 May 4]; Available from: http://databank.worldbank.org/data/download/GNIPC.pdf.

25. Sauvageot D, Njanpop-Lafourcade B-M, Akilimali L, Anne J-C, Bidjada P, Bompangue D, et al. Cholera Incidence and Mortality in Sub- Saharan African Sites during Multi-country Surveillance. [cited 2017 Aug 31]; Available from: http://pubmedcentralcanada.ca/pmcc/articles/PMC4871502/ pdf/pntd.0004679.pdf.

26. World Health Organization. Rapid Diagnostic tests. Malaria [Internet]. 2016 [cited 2016 Dec 22];1-2. Available from: http://www.who.int/malaria/areas/ diagnosis/rapid_diagnostic_tests/en/.

27. Maltha J, Gillet P, Jacobs J. Malaria rapid diagnostic tests in endemic settings. Clin Microbiol Infect. 2013:19:399-407.

28. Baluku M, Agwu E, Kasule A, Moazzam M. The transmission dynamics of cholera epidemic in Kasese district, Uganda. Spec Pathog Rev J [Internet]. 2015;1:30-9. Available from: http://www.spparenet.us/SPRJ/?page_id=194.

29. World Health Organization. Cholera vaccines: WHO position paper - August 2017. Wkly Epidemiol Rec [Internet]. 2017;2017:477-500. Available from: http://apps.who.int/iris/bitstream/10665/258764/1/WER9234-477-498.pdf.

30. Azman AS, Luquero FJ, Ciglenecki I, Grais RF, Sack DA, Lessler J. The Impact of a One-Dose versus Two-Dose Oral Cholera Vaccine Regimen in Outbreak Settings: A Modeling Study. PLoS Med. 2015;12(8).

\section{Submit your next manuscript to BioMed Central and we will help you at every step:}

- We accept pre-submission inquiries

- Our selector tool helps you to find the most relevant journal

- We provide round the clock customer support

- Convenient online submission

- Thorough peer review

- Inclusion in PubMed and all major indexing services

- Maximum visibility for your research

Submit your manuscript at www.biomedcentral.com/submit
C Biomed Central 